\title{
Neurogenesis in the hippocampus of senescence-accelerated OXYS rats in the juvenile period
}

\author{
T.A. Kozlova*, N.A. Stefanova, N.G. Kolosova, E.A. Rudnitskaya \\ Institute of Cytology and Genetics SB RAS, Novosibirsk, Russia \\ *e-mail: kozlovatanya21@gmail.com
}

Key words: neurogenesis, Alzheimer's disease, OXYS rats

Motivation and Aim: The increasing quality of life and advances of medicine in developed countries lead to increased lifespan and as a consequence growth of percentage of elderly in population. Aging is accompanied by a couple of diseases including Alzheimer's disease $(\mathrm{AD})$. In recent years it was shown, that AD-related neurodegenerative changes are associated with alterations of the neuronal plasticity. Neurogenesis is one of the major mechanisms of neuronal plasticity, and its alterations already at the young age may contribute to AD manifestation. However, the precise mechanisms of the process are not fully understood. To investigate the contribution of variations of juvenile neurogenesis to pathogenesis of AD we used OXYS rats.

Methods and Algorithms: 3-10-, 20- and 45-days-old male OXYS (model of sporadic form of $\mathrm{AD}$ ) and Wistar (control) rats were used. Immunohistochemistry was used to identify the number of neuronal cells at different stages of maturation. Development of the forelimb grasp, righting, negative geotaxis and cliff avoidance reflexes was investigated. Animal behavior and learning were evaluated in the Open field, Dark cylinder, Elevated plus maze and Morris water maze tests.

Results: OXYS pups demonstrated delayed development of negative geotaxis and righting reflexes as compared to Wistar pups. Locomotor activity of rats of both strains increased from 10 to 45 days of age; however it was lower in OXYS rats compared to Wistar rats at all analyzed ages. Moreover, 45-days-old OXYS rats demonstrated increased anxiety as well as learning and memory deficits. Behavioral and cognitive changes may reflect alterations of neuronal plasticity. Indeed, in dentate gyrus (DG) of the hippocampus of 10-day-old OXYS rats the neuronal cell density was almost twice as large as in Wistar rats due to the increased content of neuroblasts and immature neurons. From 10 to 20 days of age the neuronal cell density in DG of Wistar rats did not change while in OXYS rats it decreased. From 20 to 45 days of age the density of neuronal cells increased only in the DG of Wistar rats due to the increased density of immature neurons reflecting activation of neurogenesis in Wistar rats.

Conclusion: Delay of reflexes' development and alterations of behavior and cognitive function at young age may reflect retardation of brain development in OXYS rats. Indeed, we showed increased density of immature neurons in the DG of 10-day-old OXYS rats which may be the result of delayed neuronal maturation and/or delay of neonatal apoptosis of the neurons. Moreover, activation of neurogenesis in the DG of 45-day-old OXYS rats did not occur. These alterations of neuronal plasticity in the DG of OXYS rats occur before detection of any signs of neurodegeneration. Therefore retardation of brain development early in life may be considered as one of the risk factors associated with manifestation of AD-like pathology in adult OXYS rats.

Acknowledgements: supported by grant from the Russian Foundation for Basic Research (project no. 18-015-00320). 